\title{
Identification of genes expressed in the rat prostate that are modulated differently by castration and Finasteride treatment
}

\author{
D M Avila, S A W Fuqua ${ }^{1}$, F W George and M J McPhaul
}

Department of Internal Medicine, The University of Texas Southwestern Medical Center at Dallas, 5323 Harry Hines Boulevard, Dallas, Texas 75235-8857, USA and ${ }^{1}$ Department of Medical Oncology, The University of Texas Health Science Center, San Antonio, Texas 78284, USA

(Requests for offprints should be addressed to M J McPhaul, Department of Internal Medicine, Rm. J6·110, The University of Texas Southwestern Medical Center, 5323 Harry Hines Boulevard, Dallas, Texas 75235-8857, USA)

\begin{abstract}
In mammals, testosterone and $5 \alpha$-dihydrotestosterone (DHT) are the principal male hormones (androgens). Testosterone is the most abundant circulating androgen, and is converted in specific tissues to DHT by the $5 \alpha$-reductase enzymes. Although each of these androgens binds to the same receptor protein (androgen receptor, AR), each exerts biologically distinct effects. Theories to explain the specific effects of testosterone and DHT have centered on kinetic differences of binding of androgens to the receptor or differences in the metabolic fates of the two hormones. In the current experiments, differential display PCR (ddPCR) was used to identify genes regulated differently by testosterone and DHT. Adult male rats were treated as follows: castrated, treated with Finasteride (an inhibitor of $5 \alpha$-reductase) or left intact for ten days. RNA was prepared from the dissected prostates of these animals and used for ddPCR. Genes exhibiting four distinct patterns of regulation were observed among the mRNAs.
\end{abstract}

Class 1 genes showed equivalent expression in intact and Finasteride-treated animals, but were absent in castrated animals (mRNAs D1, D2, D6, D10). Class 2 genes showed higher expression in intact animals, intermediate levels following Finasteride treatment, but were absent in castrated animals (mRNA D8). Two classes of gene were particularly intriguing: class 3 showed gene expression only in the intact animal (mRNA D7, D9) and class 4 showed increased gene expression following Finasteride treatment (mRNA D3). While the patterns observed for some of these genes (e.g. D8) suggest that the different biological effects of testosterone and DHT may be due to the lower affinity of the AR for testosterone and limiting tissue concentrations of androgen, our results also suggest that some genes expressed in the rat prostate may be regulated in fundamentally different ways in response to testosterone and DHT.

Journal of Endocrinology (1998) 159, 403-411

\section{Introduction}

Testosterone and $5 \alpha$-dihydrotestosterone (DHT) are the principal circulating androgens in mammals and exert different actions in the male during embryogenesis and in postnatal life. Studies of animals (Goldstein \& Wilson 1972) and humans (Griffin et al. 1995, Quigley et al. 1995) with defects of the androgen receptor (AR) suggested that both hormones exert their actions via this single receptor protein. Although this receptor mediates the effects of both hormones, genetic and pharmacological data make it clear that the functions of testosterone and DHT are not equivalent. Testosterone is the principal circulating androgen in man and animals; however, the principal steroid hormone found bound to the AR in the nuclei is DHT, the $5 \alpha$-reduced metabolite of testosterone (Bruchovsky \& Wilson 1968, Grino et al. 1990a). Genetic studies of individuals defective in the synthesis of one of the two enzymes that catalyze this conversion ( $5 \alpha$-reductase 2 ) provides further evidence that this biochemical transfor- mation is physiologically important (Russell \& Wilson 1994). Patients deficient in this enzyme show a phenotype characterized by female external genitalia and the absence of prostatic development, but with normal virilization of the male Wolffian duct-derived internal organs. Equally important, pharmacological studies have demonstrated that the administration of specific inhibitors of the $5 \alpha-$ reductase enzyme(s) in animals during development causes defects of phenotypic male development that mirror the defects of virilization observed in patients with $5 \alpha-$ reductase type II deficiency (Imperato-McGinley et al. 1985, George \& Peterson 1988).

At least three mechanisms might account for the differential actions of these hormones. First, each hormone might have a different allosteric effect on the receptor and thus promote the modulation of different but overlapping sets of androgen responsive genes. Secondly, the formation of DHT in some tissues might be required to enhance a weak signal exerted by testosterone that is present in limiting concentrations (signal amplification). In this latter 
theory, testosterone in high concentrations could perform all androgen actions, and DHT formation would be most important in tissues with low androgen content. This latter theory could explain the fact that exogenous DHT can virilize all portions of the urogenital tract in the female embryo (Schultz \& Wilson 1974). Finally, the metabolic conversions of testosterone and DHT are not equivalent. Testosterone can be aromatized to estrogen, while DHT cannot. Such differences might allow for the selective regulation of genes by testosterone or DHT owing to selective conversion to metabolites (such as estrogen).

In previous studies, we demonstrated that a model androgen responsive gene, MMTV-CAT, is regulated in cells deficient in androgen metabolizing enzymes in a way that is most consistent with $5 \alpha$-reductase performing an amplifying function. That is, although the maximum reporter gene activation by testosterone and DHT was the same, differences were evident in the kinetics of gene activation effected by testosterone and DHT (Deslypere et al. 1992). Although these studies established the existence of one type of regulation of genes by testosterone and DHT, they offered no information regarding the relevance of these findings to a larger group of androgenresponsive genes in androgen target tissues. The current studies seek to examine these alternative models of gene regulation by testosterone and DHT in a more general fashion. To this end, we have used differential display PCR to identify genes that are expressed in the rat prostate in intact (normal levels of testosterone and DHT), castrated (low levels of testosterone and DHT), and Finasteride-treated (low levels of DHT) animals in patterns that suggest differential control by testosterone or DHT.

\section{Materials and Methods}

\section{Treatment protocols and $R N A$ extraction}

Twenty mature male rats (each weighing approximately $200 \mathrm{~g}$ ) were treated as follows: three were kept intact, ten were castrated and seven were treated with Finasteride $(50 \mathrm{mg} / \mathrm{kg} /$ day s.c. in $20 \%$ ethanol in triolein oil). Following the treatment period, the rats were killed and the prostates were dissected, weighed, and used to prepare RNA. RNA was isolated using the Ultraspec RNA isolation kit (Biotecx Laboratories, Inc., Houston, TX, USA). The wet weights and RNA yields from each of the treatment regimens are shown in Table 1. As reported previously (George 1997), the concentration of DHT was reduced by $90 \%$ in the prostates of animals treated with Finasteride.

\section{Differential display PCR}

The display PCR protocol used in these studies is derived from that described by Liang and Pardee (1992). cDNA was synthesized from total rat prostate RNA using 1-2 $\mu \mathrm{g}$ total RNA in $10 \mathrm{mM}$ Tris- $\mathrm{HCl}$ (pH 8.3), $50 \mathrm{mM} \mathrm{KCl}$, $3 \mathrm{mM} \mathrm{MgCl} 2,0.01 \%$ gelatin, $1 \mathrm{nmol}$ each of the primers $\left(\mathrm{T}_{11} \mathrm{CA}, \mathrm{T}_{11} \mathrm{GG}, \mathrm{T}_{11} \mathrm{GC}\right.$, and $\left.\mathrm{T}_{11} \mathrm{AT}\right)$, and $800 \mu \mathrm{M}$ of four deoxyribonucleoside triphosphates (dNTPs). The reactions were incubated at $94{ }^{\circ} \mathrm{C}$ for $2 \mathrm{~min}$, cooled, and the reverse transcription initiated by the addition of 2 units AMV reverse transcriptase (AMV-RT; Promega, Madison, WI, USA) and incubation at $40{ }^{\circ} \mathrm{C}$ for $1 \mathrm{~h}$. Following this period, the reactions were incubated at $70{ }^{\circ} \mathrm{C}$ for $10 \mathrm{~min}$ and $94{ }^{\circ} \mathrm{C}$ for $5 \mathrm{~min}$. Two units Ribonuclease $\mathrm{H}$ (Gibco BRL, Gaitherburg, MD, USA) were added to the reactions and the incubation continued at $37^{\circ} \mathrm{C}$ for an additional $1 \mathrm{~h}$. The reactions were then incubated at $94{ }^{\circ} \mathrm{C}$ for $5 \mathrm{~min}$ and either kept at $-20{ }^{\circ} \mathrm{C}$ or used in the PCR reaction.

The cDNAs resulting from these reactions were then amplified by low stringency PCR using the previously described buffer, $1 \mathrm{nmol}$ each of the primers described above, $1 \mathrm{nmol}$ each of the $10 \mathrm{bp}$ random primers (CTGATCCATG, CCAACGATGC, and AGAACAG TCA $)$ and $800 \mu \mathrm{M}$ of the dNTPs. $\left[{ }^{32} \mathrm{P}\right] \mathrm{dCTP}(15 \mu \mathrm{Ci}$, $3000 \mathrm{Ci} / \mathrm{mmol}$; Amersham, Arlington Heights, IL, USA) was included to label the product of the PCR reaction. Native Taq polymerase (2.5-5 units; Perkin Elmer, Branchburg, NJ, USA) was added to the reactions followed by a 2 min incubation at $94^{\circ} \mathrm{C}$. Each PCR cycle consisted of a $30 \mathrm{~s}$ denaturation at $94{ }^{\circ} \mathrm{C}$, followed by $1 \mathrm{~min}$ annealing at $42^{\circ} \mathrm{C}$ and $30 \mathrm{~s}$ extension at $72{ }^{\circ} \mathrm{C}$. After 40 cycles, the final products were extended for $5 \mathrm{~min}$ at $72{ }^{\circ} \mathrm{C}$ with subsequent incubations of $10 \mathrm{~min}$ at $98^{\circ} \mathrm{C}$ and $15 \mathrm{~min}$ at $27^{\circ} \mathrm{C}$. The amplified cDNAs were precipitated with ethanol from $0.3 \mathrm{M}$ sodium acetate, resuspended, and counted. Approximately 16000 counts per minute per sample were applied to a 6\% Long Ranger (AT Biochem, Malvern, PA, USA) gel cast using $1 \times$ TBE. The RTS label-ready 100 bp DNA ladder from Gibco BRL was included in each gel as size standard. After electrophoresis, the gels were dried and exposed to X-ray film.

\section{cDNA reamplification cloning and sequencing}

After comparing the patterns visualized by autoradiography, individual bands were selected for study. This selection was based on apparent decreases in expression in response to castration or treatment with Finasteride. The gel fragments corresponding to these bands were cut out and eluted in water after heating at $68^{\circ} \mathrm{C}$ for $1 \mathrm{~h}$. The individual cDNA fragments were reamplified using aliquots of the eluted gel slices as template using the same primers and amplification parameters as those used to produce them through either display PCR or RT-PCR (40 cycles). The amplified products were purified by electrophoresis on agarose gels and cloned using the TA cloning vectors (InVitrogen, San Diego, CA, USA). 
Clones were isolated with the Qiagen plasmid isolation kit (Chatsworth, CA, USA) and those containing inserts were sequenced using the DNA Sequenase kit from US Biochemical Corp. (Cleveland, OH, USA).

\section{High stringency $R T-P C R$ reactions}

The RT-PCR reactions consisted of obtaining a specific cDNA from RNA using specific primers and amplification of such cDNA by PCR. In these reactions, cDNA was prepared by priming 1-2 $\mu \mathrm{g}$ total prostate RNA with $1 \mathrm{nmol}$ of a specific $\mathrm{T}$-tailed primer using the previously described AMV-RT reaction conditions. The resulting cDNA was used in a high stringency RT-PCR amplification reaction using a specific set of oligonucleotide primers (25 nucleotides in length) derived from the nucleic acid sequence of the individual cDNAs. Although the high stringency reactions employed the same buffer conditions as described above, the amplification conditions differed considerably. The reactions were incubated at $95^{\circ} \mathrm{C}$ for $5 \mathrm{~min}$ and at $70{ }^{\circ} \mathrm{C}$ for $5 \mathrm{~min}$. One unit Perfect Match (Stratagene, La Jolla, CA, USA) and 2.5-5 units native Taq polymerase were then added to the reactions followed by a $2 \mathrm{~min}$ incubation at $94{ }^{\circ} \mathrm{C}$. The PCR cycle of amplification consisted of $30 \mathrm{~s}$ denaturation at $94{ }^{\circ} \mathrm{C}$, followed by $30 \mathrm{~s}$ annealing at $70^{\circ} \mathrm{C}$ and $1 \mathrm{~min}$ extension at $72{ }^{\circ} \mathrm{C}$. After 25 cycles the final products were extended, as previously described. For C3 and AR RT-PCR reactions the following primers were used: CTGGCTGCAGTAT TCTAGATGAAG (C3 sense), GGCTGTTGACAGCT TTCACTGTTA (C3 antisense) and CATGAAAGCA CTGCTACTCTTCAG (AR sense), GCTCACCATA TGGGACTTGATTAG (AR antisense) respectively. The cDNAs were precipitated and applied to a $6 \%$ sequencing PAGE cast using $1 \times$ TBE buffer. After electrophoresis the gels were dried and exposed to X-ray film.

\section{Results}

\section{Treatment protocols}

In these studies, RNA was prepared from prostate tissue that was exposed to a range of hormonal environments: stimulation by DHT and testosterone (intact male rats), predominantly testosterone (Finasteride-treated male rats), or by neither DHT nor testosterone (castrated male rats). As outlined previously (George 1997), under the conditions used in this Finasteride treatment regimen, prostatic DHT concentrations fell by $90 \%$, while intraprostatic testosterone concentrations rose 3- to 5-fold. Of note, each of the treatment regimens used in these experiments was continued for a total of ten days. This protocol was designed to minimize the contribution from acute changes in gene expression caused by the changes in cellular composition that occur in the prostate immediately following androgen withdrawal or administration.
Table 1 Average prostate weights (means \pm S.D.) and RNA yields of prostates from intact, castrated and Finasteride-treated intact rats

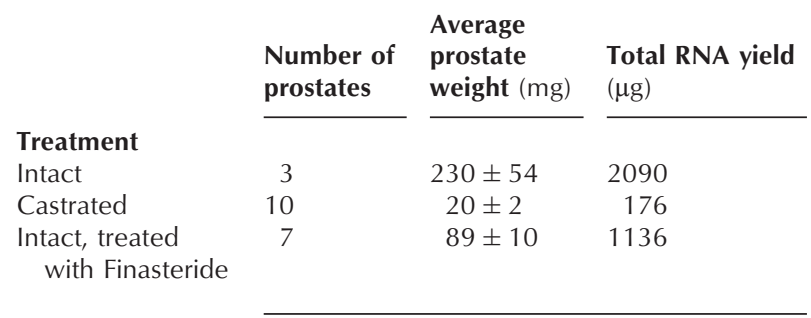

The characteristics of the harvested tissues and RNA yields in one such experiment are shown in Table 1.

\section{Differential display PCR and RT-PCR}

Differential display PCR was used to analyze these RNA samples using 12 different combinations of oligonucleotide primers, as outlined above. A portion of a representative gel from one such experiment is shown in Fig. 1. Although very large numbers of changes are evident between the different samples in experiments using each primer set, our analysis centered on identifying mRNAs that appeared to reflect potential differences of regulation by testosterone and DHT. In this selection process, we focused our efforts on those genes that exhibited decreased expression in response to the two forms of androgen deprivation. Portions of the dried gel corresponding to the bands of interest were cut from the gel, eluted, reamplified, cloned and sequenced. A total of 25 possible candidates were eluted and 14 of these could be amplified. Of these 14 fragments, 8 partial cDNAs were cloned and characterized in detail. A summary of the characteristics of these clones and their patterns of regulation as inferred from the differential display PCR gel are shown in Table 2. The derived nucleotide sequences of the partial cDNA clones are presented in Table 3 .

The level of expression of these clones as visualized on the display PCR gels suggested that we have isolated clones that are expressed at different levels under the different treatment conditions. As the display PCR experiments employed degenerate primers and low stringency conditions it was possible that the changes observed were a reflection of changes in the overall cellular composition of expressed RNAs and might not accurately reflect the abundance of the specific mRNAs under study. For this reason, the expression levels of each of the clones was examined further using a high stringency RT-PCR assay that employed specific primers derived from the nucleotide sequence of each clone. In these experiments, the specific primers were derived from the segments $5^{\prime}$ to the poly A tail and distinct from those used in the original display PCR. Representative gels from such experiments are shown in Fig. 2 and the results of all such experiments 


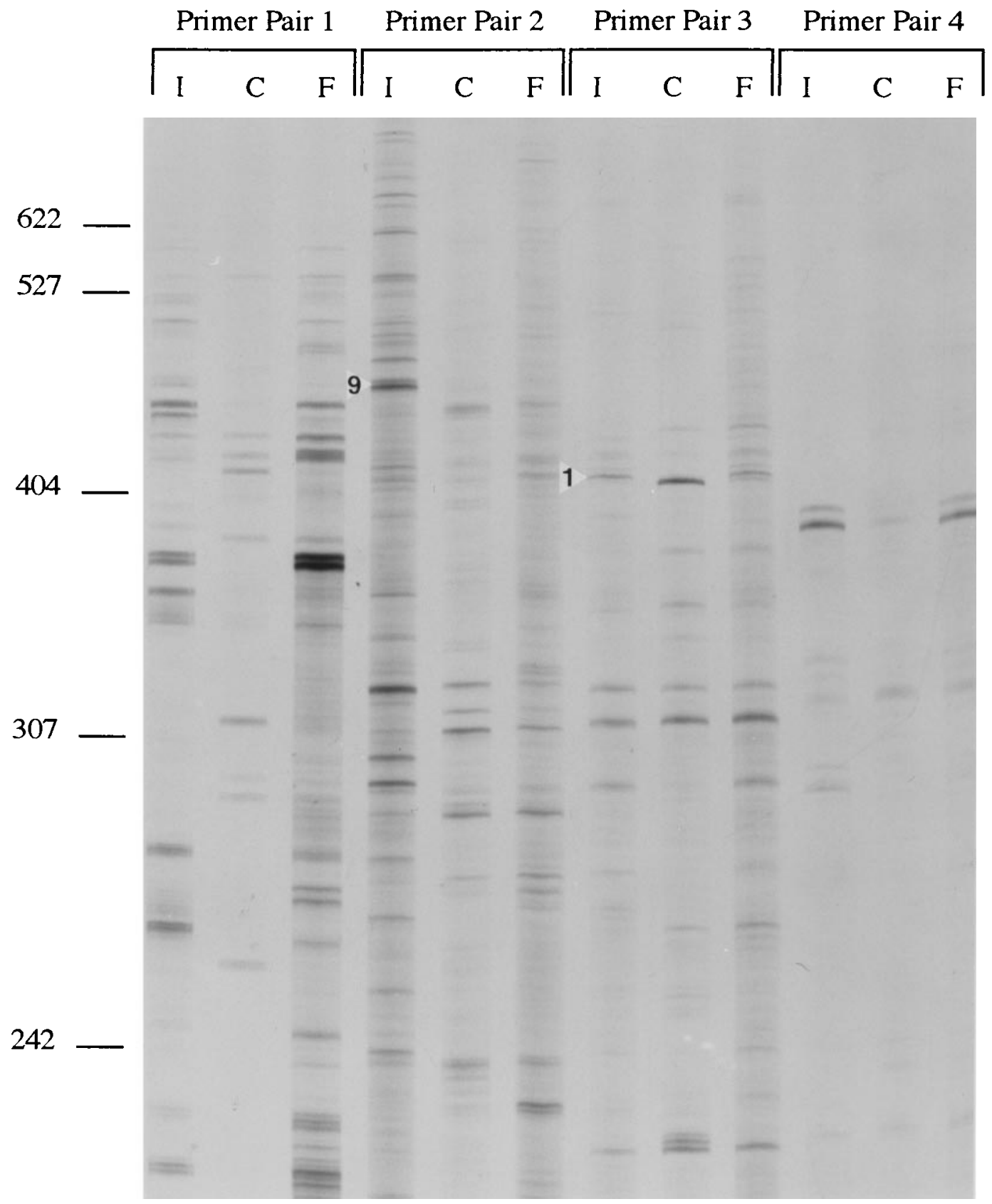

Figure 1 Differential display PCR of mRNAs expressed in rat prostates dissected from intact male rats (I), castrated male rats (C), and intact male rats treated with Finasteride $(\mathrm{F})$. Similar quantities of labeled cDNA (approximately $1.6 \times 10^{5}$ disintegrations per minute) were loaded in each lane and electrophoresed on a $6 \%$ denaturing polyacrylamide gel. Following electrophoresis, the gels were dried and autoradiographed. The autoradiograph shows the analysis of the same RNA preparations using four different primer pairs (pair 1:

$\mathrm{T}_{11}$ CA/AGAACAGTCA; pair 2: $\mathrm{T}_{11} \mathrm{GG} / \mathrm{CTGATCCATG;} \mathrm{pair} \mathrm{3:} \mathrm{T}_{11} \mathrm{GG} / \mathrm{CCAACGATGC}$; pair 4: $\mathrm{T}_{11} \mathrm{GG} /$ AGAACAGTCA). The white arrows indicate the position of the amplified bands corresponding to clones D9 and D1 in the differential display autoradiograph.

are tabulated in Table 2. The results indicate that the patterns of expression inferred from the results of the low stringency differential display PCR reactions were accurate and only minor discrepancies were evident compared with the results of the high stringency RT-PCR reactions. 
Table 2 cDNA clone characteristics and patterns of regulation

\begin{tabular}{|c|c|c|c|c|c|c|c|}
\hline \multirow[b]{2}{*}{ Clone } & \multicolumn{3}{|c|}{$\begin{array}{l}\text { Pattern observed in } \\
\text { display PCR experiments }\end{array}$} & \multicolumn{2}{|c|}{ cDNA fragment } & \multirow[t]{2}{*}{$\begin{array}{l}\text { Pattern observed in } \\
\text { high stringency RT-PCR }\end{array}$} & \multirow[t]{2}{*}{ Pattern type } \\
\hline & I & $\mathrm{C}$ & $\mathrm{F}$ & Size (bp) & Unique & & \\
\hline D1 & + & - & + & 359 & Yes & Same ${ }^{a}$ & 1 \\
\hline D2 & $2+$ & $+1-$ & $2+$ & 289 & Yes & Same ${ }^{a}$ & 1 \\
\hline D3 & + & + & $3+$ & 303 & Yes & Same ${ }^{a}$ & 4 \\
\hline D6 & $2+$ & - & + & 211 & Yes & $1+-2+^{a}$ & 1 \\
\hline D7 & + & - & - & 224 & Yes & Same ${ }^{a}$ & 3 \\
\hline D8 & $2+$ & - & + & 225 & Yes & Same ${ }^{a}$ & 2 \\
\hline D9 & + & - & - & 479 & Yes & Same ${ }^{a}$ & 3 \\
\hline D10 & + & - & + & 293 & Yes & Same ${ }^{a}$ & 1 \\
\hline
\end{tabular}

The patterns of expression of 8 different cDNA clones as visualized by display PCR are indicated on the left using a semiquantitative scale $(-($ mRNA not detected $),+($ least $)$ to $3+($ most $))$ to grade the intensity of bands observed. The characteristics of the isolated cDNA fragments are presented in the center. The pattern of expression inferred from 'hot' RT-PCR experiments using specific primers (based on the insert nucleotide sequence) and stringent PCR conditions is shown to the right. Superscript a indicates that the bands visualized in the hot PCR reaction have been shown to have the expected structure by sequence analysis of bands isolated from the RT-PCR reactions. I, intact rats; C, castrated rats; F, Finasteridetreated intact rats.

\section{Discussion}

Testosterone and DHT are the major circulating androgens in human and in other vertebrates. Although each hormone displays potent effects in biological assays of androgen action (e.g. prostate growth), under specific conditions each hormone displays properties that suggest roles in the modulation of specific functions. It is clear that DHT is crucial for the normal development of the male external genitalia and of the prostate (Imperato-McGinley et al. 1985, George \& Peterson 1988). In contrast, testosterone appears to play essential roles in promoting spermatogenesis and Wolffian duct development, as well as gonadotropin regulation.

Although several potential explanations could account for the different biological effects of testosterone and DHT, three models seem most plausible. In the first model, both testosterone and DHT are capable of regulating androgen responsive genes to the same maximal extent and the biological differences evident between the activities of the two hormones would be due to kinetic differences in the binding of testosterone and DHT by the $\mathrm{AR}$ and limiting concentrations of testosterone and DHT at the cellular level (Veyssiere et al. 1982, Grino et al. 1990b). In this scenario, even in intact animals the tissue concentrations of androgens are below those needed to saturate the AR. In this context, the formation of DHT which is more avidly bound by the receptor-would explain the dependence of some phenomenon on the formation of DHT. A second model postulates that although many genes can be regulated both by testosterone and DHT, the conformation of the two receptor-hormone complexes are not identical and that these differences confer specific regulation by only testosterone-AR or DHT-AR complexes on selected genes (Fang et al. 1969). Such selective regulation of genes by the two androgens has not yet been described. Finally, differences in the metabolism of DHT and testosterone might account for their different biological effects. In this model, metabolic transformations of testosterone and/or DHT would lead to the formation of metabolites that possess unique biological properties. Although the aromatization of testosterone to form estradiol is the most obvious example, other transformations could be involved.

To date, the only work to examine the mechanisms by which testosterone and DHT act to regulate androgenresponsive genes has been performed using a model androgen-responsive gene, MMTV-CAT (Deslypere et al. 1992). Although the results of this study clearly demonstrate that this model gene is regulated by androgen in a manner most consistent with the first model described above, it has not been clear how representative these results are compared with the larger number of androgenregulated genes expressed in androgen target tissues. Furthermore, while a number of studies have used differential display to examine the differential expression of genes in prostate tissue or prostatic cells (see, for example, Blok et al. 1995 or Chapman et al. 1995), only a single report (Lin \& Chang 1997) has reported the isolation of genes differentially regulated by testosterone and DHT.

The current study was designed to begin to address these questions in a more general fashion using a tissue (the rat prostate) in which the importance of DHT formation is well established (Imperato-McGinley et al. 1985, George \& Peterson 1988). Three features have been incorporated into this study to simplify the analysis of results. First, to minimize changes in gene expression caused by fluctuations in hormone levels (e.g. following androgen administration to castrated animals), we have compared mRNAs expressed in the rat prostate following no treatment 
Table 3 Sequences of cDNA fragment clones isolated from differential display PCR

\begin{tabular}{ll} 
Clone & \multicolumn{1}{l}{ sequence } \\
D1 & GCTTTTGAGAAATATATCGGGTAAGGTTGAAAAATTAGACCTGAATA \\
& AATTCATTAATACAGAACCATACAGTGAAGACGATAAAATTTATTGCGGCA \\
ATTAAGAAAATATTGCCTAATGAAATAACAAAGTATTTAAACCGTAATCTC \\
CGTTCAGTGGTTTTGGGAAAACATTATTCAACAGCATGATGATGAGCTACC \\
TGAAGAAACCAGAAAGCTGATTAACGAATACCTTCACCCCAAAATAAAAAAA \\
ATATTAGCAGAATTAAGTGAAAGCAATTTGCATTTATCTGCCGGATAAAA \\
AATCTTTACAACTGCTAACTCACAAAAAGCTGAATTATTGCAGAGCCTGC \\
TGTGCCTGAGTTTACGTTAATATGCTAATGCTAAGTATGAGATTGAATGT \\
AAGGTAAAGGCATCC(A) 11 \\
D2 \\
CGTCTTGCCGTATGCAGTTGTATTATTTCCTCAACTGTAATGTTTTTGATTTA \\
CTCACTTCTTCTAAATCAATACCAAAAGAAATATCATAGCAAACAGGAATTT \\
GTATGACTTTTTTTCTTGCGTCATTCGAGATGGGGTTCTTGCTCAACGAG \\
AAATCTCTCGAATTTATTAAGACAAGATTTTCTGCACGAACCTCGAAATGA \\
CGATTGATTCTTTACCGCATTTCAATTGCTCTTTATAAATTGATAAGGT \\
TGGGAATGCTTTGATAAATAACATAAGCATCGTCG(A) 11
\end{tabular}

D3 CCGGCAGACATTGACATCTCTTCCATAGTACAGCCCTTGGCTGCAGA GACTCCTCAGATCTGAGAGGCTGAACAGAGACTGGTAATATGCGGCCAGC GAAGGGTCATCTCTCTGAATGAGAAGGGGCTTCTGTTCCCAGAATTCCTTG AAAAATGTCTCTACTTTGATGGGTGATATTAAACTTTCAAAGAAACTACTGG GGCTGTCAAAGTTTAATACAGAAAGCGTATTAGGTAGCTCCTCTTTCACCTG CTTGCAAGGAACAGACTCTTCTTCATTCTCATCCCCTGTGGGCTTCACTTTCT TTGGCATCGTTGGCG(A) 11

D6 GAAATGAAAGAACTGGCTCAACTTCTCCAAGATTCTAGTAAGACTTTT GATTAAAATTATTCCATTCTTTTTACAGGCTCTAGATCTTTTAATTCTTCTTCA GTAAAAAGTCTGTAAATAATTTTAAAAGTCTTTCCTAATGGTGTTTCCAGAGA AAACCCGCCTGGTCCGAAACCGTCTGTAACATCTAGCGTGAAATGTGAAAA TTTCCAGTATTCAAATAAATCTCTATCAATCCAAAATTCAAAACCATCTACCA AACCAATCATAGCATCGTTGGCC(A) 11

D7

CATTGATGCCAGGAGCCTTCCCTGGTCATACTTCATGCAACCACTCT ACGATTATACATAGATTTCACTGTAGTTTATGTGTGAATCCACTTTCTCTAAG GGTCTAATCACTGAACAAGCTACAACCAAAACATAGCCATTGTCCCAAGTTT TATAAACTGTATATGCCGTTTTATTGTTAATTGAATTGACTAATAGACAAGTC ATTTATTCTACATGTACATG(A) 11

(testosterone and DHT present), castration (neither present), or treatment with Finasteride, an inhibitor of $5 \alpha$-reductase (predominantly testosterone present). It is important to note that the protocol employed for the administration of Finasteride in this study has been previously described and shown to result in defined levels of 
Table 3 Continued

\begin{tabular}{ll} 
Clone & \multicolumn{2}{l}{ Sequence } \\
\cline { 2 - 2 } D8 & GTAATAATAAAACATTCTTCTCAGATGACTTCTTGCACACACAAAAAT \\
& GATTCTGCAAGCACAACAGAACTTATTCCAAGTTAAGCACTGTGTGTACCA \\
AAAGAAAAAGAAAATTATCATTCAATAAACCCATTCATTATTTTTCTAAAGA \\
CTAAGAACTGATTCCTTTCATCAATTCATTAATGTTAACACTTTTAAAAATC \\
AGATTTACAATTCCATAACAGGTCTGCGTG $(A)_{11}$
\end{tabular}

D9

CCAACGATGCGGAACCACTGAAGTACATGGTGGGTTTTAGAAATCTC AGTATTTCTTTACCTAGATACTTGCCCAATGTGAGAACTGTACCAGATGTCA GCCTCTGTATGTCCTCACTATTCTCCCACTTGTCTTTCCTCTGTTTATTCATTC ACATGGCTCCAGGCCTGGTTCTCGTCTCATTTTCCTTGATGTTTAATTTTTAT TTGTTTAACTATTAATATTTATAATTAATATTTATCTGTTTATTCTGCTAACTTT TATTCTTTAATTTTTCATTTTTGTTTTGACAACTCTGGTTTTTTATTTGGTGCAT CGTTGGCATGCATGCACCAACGATGCGGAACCACTGAAGTACATGGTGGGT TTTAGAAATCTCAGTATTTCTTTACCTAGATACTTGCCCAATGTGAGAACTGT CCAGATGTCAGCCTCTGTATGTCCTCACTATTCTCCCACTTGCTTTCCTCTGT TATTCATTCACATGGCTCCAGGCCTGGTCTCGTCTCATTTTCCTTGATGTTTA ATTTTATTTGTTAACTATTAATATTTATAATTAATATTTATCTGTATTCTGCTAA CTTTTATTCTTTAATTTTCATTTTTGGTTTGACAACTCTCC(A)

D10

GGTGAAACAGAGGCAAAGCACGTGGTTACAGCTCTAGAGCTGGCAA
GCGGCAAAATACCAAGGGGCAACTGGAAACCTATGGCAGGGTTTGTAAGG
AGATGGCCAAGGTCTTAAACATGTCAGACTGAGAAAATGGAAGGCCAAAGA
GGGATTTAAAAAGAGAGATTGACGGGGTTGGGGATTTAGCTCAGCGATAG
AGCGCTTGCCTAGGAAGCGCAAGGTCCTGGGTTCGGTCCCCAGCTCCGAA
AAAAAGACCCAAAAAAAAAAAAAAAGAGAGAGACAGAGAGGTTGACACTT
GCAACATACAAAACAACATGACATGAGTGTGTGTGTGCACATTTGTGTGTGT
ATGAGCGACTGTGTGTATGAGAGAGAAAGTGTGTGTGTGAGAGAGTGTAT
GTGTGTATGTGAGTGACTGTTCTG(A) ${ }_{11}$

The nucleotide sequence of each of the cloned cDNA fragments is presented. In each instance, the segment depicted is followed by repeated adenosine residues derived from the poly-A tail. The clone designations are those defined in Table 2. The specific primers used for high stringency RT-PCR of each clone are underlined.

testosterone and DHT in serum and in the prostate itself (George 1997). In the prostates of animals treated in this fashion, DHT levels were reduced by $90 \%$, while testosterone levels increased 3- to 5-fold. Secondly, we have attempted to avoid the contributions of the well known changes in the cell composition of prostate that occur following castration and androgen administration in these studies by using a 10 -day treatment regimen prior to tissue harvesting. This has been done in order to minimize changes in mRNA directly related to alterations in the tissue composition, caused by either apoptosis or regrowth. Finally, we have focused our attention on identifying genes that show increased expression in the intact or
Finasteride-treated animals, compared with the castrated rats. Although this condition certainly will result in the exclusion of genes regulated negatively by androgen from our study, this criterion is also likely to exclude genes that appear to increase in abundance following castration only as a result of the large scale changes occurring in the level of highly expressed RNAs in the prostate.

The eight genes isolated and analyzed in this study display four different patterns of regulation. Pattern types class 1 (D1, D2, D6, D10) and class 2 (D8) all show detectable expression of transcript in the RNA prepared from both the intact and Finasteride-treated animals (see Table 2). Both patterns are consistent with model 1 (see 


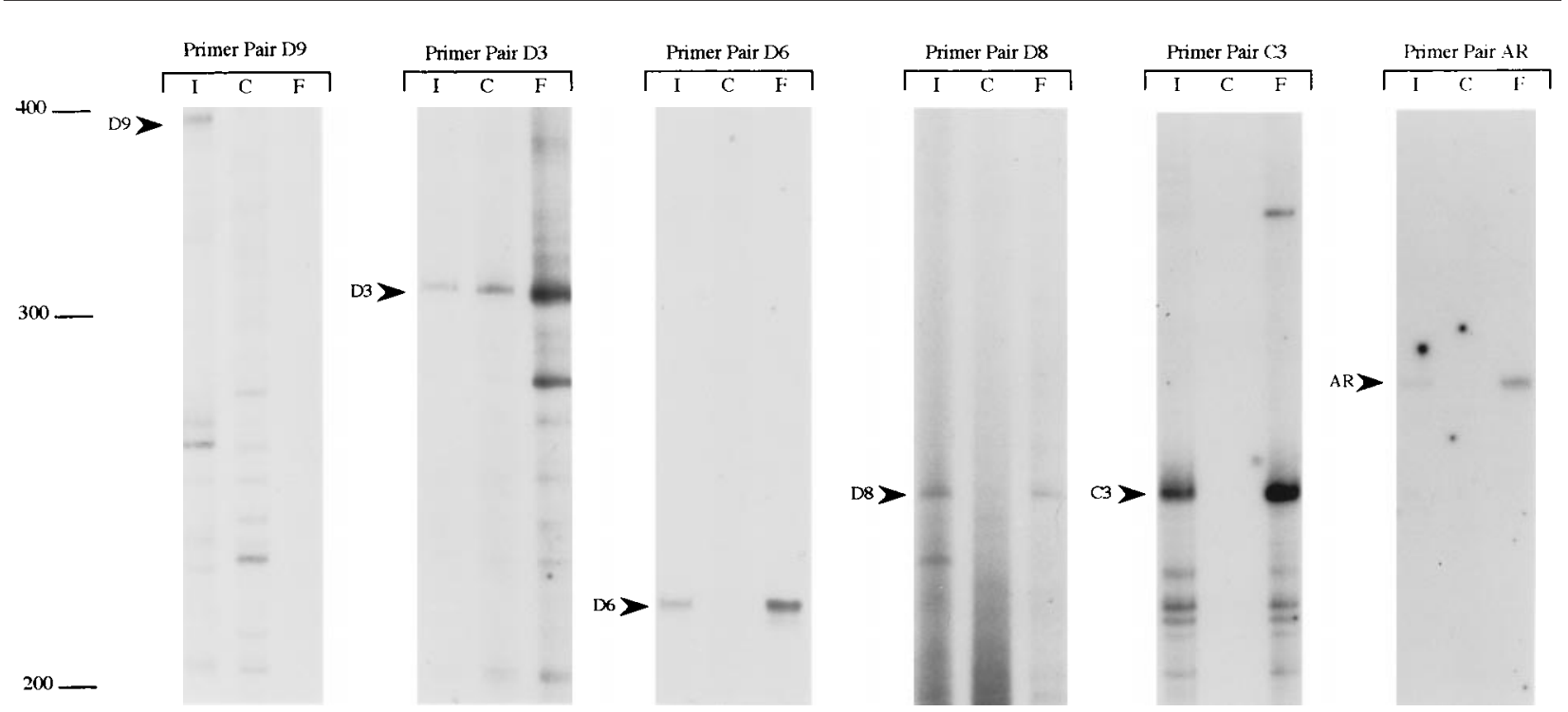

Figure 2 RT-PCR of specific mRNAs expressed from rat prostates from intact male rats (I), castrated male rats (C) and intact male rats treated with Finasteride (F). The autoradiograph shows the analysis of the same RNA preparations using four sets of specific primers. The arrows indicate the positions of the amplified bands corresponding to fragments of the D9, D3, D6, and D8 mRNAs (identities of these bands have been proven by elution of the visualized bands from the gel, reamplification, and nucleotide sequence analysis). This autoradiograph demonstrates the four different patterns of mRNA expression that we have encountered. D9, pattern 3; D3, pattern 4; D6, pattern 1; D8, pattern 2. For comparison, assays of AR and C3 mRNA in these same RNA specimens using RT-PCR assays are shown in the two right-hand panels.

above), in which differences in the level of expression of genes in response to testosterone and DHT could be accounted for on the basis of differences in the kinetics of testosterone and DHT binding by the AR and limiting androgen concentrations. Together these two patterns account for over $50 \%$ of the genes that we have studied in detail (Table 2) or by inspection of our original display PCR gels (see Fig. 1).

In contrast, genes displaying the pattern termed class 3 (cDNAs D7 and D9) are detected only in RNA prepared from the prostates of intact animals. While these genes are candidates to be genes that are only regulated in response to $5 \alpha$-dihydrotestosterone, it is also possible that these genes are exhibiting the same type of pattern observed for clone D8, but with a level of expression in the Finasteridetreated animals that is below the limits of sensitivity of the assay used. Further studies will be required to discriminate between these two possibilities.

The pattern observed for gene D3 (class 4) is quite unexpected, with the highest levels of expression being observed in RNA prepared from the prostates of Finasteride-treated animals. Although this pattern could be potentially explained in a number of different ways, selective regulation by testosterone or one of its metabolites (e.g. estradiol or androstenediol) or by the increased intraprostatic levels of testosterone that result from the Finasteride treatment regimen seem to be the most likely. Of interest, two mRNAs that are known to be regulated by androgen - those encoding $\mathrm{C} 3$ and the $\mathrm{AR}$ - exhibit this pattern of regulation in RT-PCR assays.

In summary, we report the isolation and characterization of partial cDNAs representing genes that show four different patterns of regulation in response to castration and Finasteride treatment. Although it is impossible to predict whether any of the genes that we have identified will show absolute or selective regulation by testosterone or DHT, it is clear that we have identified genes that show substantially different patterns of androgen regulation in castrated and Finasteride-treated animals. While the patterns observed for many of these genes suggest that the different biological effects of testosterone and DHT may be due to the lower affinity of the AR and limiting tissue concentrations of androgen, our results also suggest that some genes expressed in the rat prostate may be regulated in fundamentally different ways in response to testosterone and DHT. Further studies will be required to identify the sites of expression of these genes within the prostate and the mechanisms that are responsible for these apparent differences in expression in response to testosterone and DHT.

\section{Acknowledgements}

This work was supported by grants DK52678 (MJM) and CA30195 (SAWF) from the National Institutes of Health. 


\section{References}

Blok LJ, Kumar MV \& Tindall DJ 1995 Isolation of cDNAs that are differentially expressed between androgen-dependent and androgen-independent prostate carcinoma cells using differential display PCR. The Prostate 26 213-224.

Bruchovsky N \& Wilson JD 1968 The intranuclear binding of testosterone and $5 \alpha$-androstan-17 $\beta$-ol-3-one by rat prostate. Journal of Biological Chemistry 243 5953-5960.

Chapman MS, Qu N, Pascoe S, Chen WX, Apostol C, Gordon D \& Miesfeld RL 1995 Isolation of differentially expressed sequence tags from steroid-responsive cells using mRNA differential display. Molecular and Cellular Endocrinology 108 R1-R7.

Deslypere J-P, Young M, Wilson JD \& McPhaul MJ 1992 Testosterone and $5 \alpha$-dihydrotestosterone interact differently with the androgen receptor to enhance transcription of the MMTVCAT reporter gene. Molecular and Cellular Endocrinology 88 15-22.

Fang S, Anderson KM \& Liao S 1969 Receptor proteins for androgens. Journal of Biological Chemistry 244 6584-6595.

George FW 1997 Androgen metabolism in the prostate of the Finasteride-treated adult rat: a possible explanation for the differential action of testosterone and $5 \alpha$-dihydrotestosterone during development of the male urogenital tract. Endocrinology 138 871-877.

George FW \& Peterson KG 1988 5 $\alpha$-Dihydrotestosterone formation is necessary for embryogenesis of the rat prostate. Endocrinology 122 1159-1164.

Goldstein JL \& Wilson JD 1972 Studies on the pathogenesis of the pseudohermaphroditism in the mouse and testicular feminization. Journal of Clinical Investigation 51 1647-1658.

Griffin JE, McPhaul MJ, Russell DW \& Wilson JD 1995 The androgen resistance syndromes. Steroid $5 \alpha$-reductase 2 deficiency, testicular feminization, and related disorders. In The Metabolic and Molecular Bases of Inherited Disease, edn 7, pp 2967-2998.
Eds CR Scriver, AL Beaudet, WS Sly \& D Valle. New York: McGraw-Hill.

Grino PB, Griffin JE \& Wilson JD 1990a Androgen resistance due to decreased amounts of androgen receptor: a reinvestigation. Journal of Steroid Biochemistry 35 647-654.

Grino PB, Griffin JE \& Wilson JD $1990 b$ Testosterone at high concentrations interacts with the human androgen receptor similarly to dihydrotestosterone. Endocrinology 126 1165-1172.

Imperato-McGinley J, Binienda Z, Arthur A, Mininberg DT, Vaughan ED Jr \& Quimby FW 1985 The development of a male pseudohermaphroditic rat using an inhibitor of the enzyme 5a-reductase. Endocrinology 116 807-812.

Liang P \& Pardee AB 1992 Differential display of eukaryotic messenger RNA by means of the polymerase chain reaction. Science 257 967-971.

Lin TM \& Chang C 1997 Cloning and characterization of TDD5, an androgen target gene that is differentially repressed by testosterone and dihydrotestosterone. Proceedings of the National Academy of Sciences of the USA 94 4988-4993.

Quigley CA, De Bellis A, Marschke KB, el-Awady MK, Wilson EM \& French FS 1995 Androgen receptor defects: historical, clinical, and molecular perspectives. Endocrine Reviews 16 271-321.

Russell DW \& Wilson JD 1994 Steroid $5 \alpha$-reductase: two genes/two enzymes. Annual Review of Biochemistry 63 25-61.

Schultz FM \& Wilson JD 1974 Virilization of the Wolffian duct in the rat fetus by various androgens. Endocrinology 94 979-986.

Veyssiere G, Berger M, Jean-Faucher C, De Turchkheim M \& Jean C 1982 Testosterone and dihydrotestosterone in sexual ducts and genital tubercle of rabbit fetuses during sexual organogenesis: effects of fetal decapitation. Journal of Steroid Biochemistry 17 149-154.

Received 20 October 1997

Revised manuscript received 10 June 1998 Accepted 4 August 1998 